\title{
Lentivirus shRNA Grb10 targeting the pancreas induces apoptosis and improved glucose tolerance due to decreased plasma glucagon levels
}

\author{
B. Doiron • W. Hu • L. Norton • R. A. DeFronzo
}

Received: 30 August 2011 / Accepted: 24 November 2011 /Published online: 7 January 2012

(C) Springer-Verlag 2012

\begin{abstract}
Aims/hypothesis The physiological significance of growth factor receptor-bound protein-10 (GRB10) in the pancreas is unclear. We hypothesised that GRB10 is involved in pancreatic apoptosis, as GRB10 binds with a family of cell-survivalrelated proteins implicated in apoptosis.

Methods Lentiviral vector small hairpin RNA (shRNA) targeting Grb10 was injected in vivo via an intraductal pancreatic route to target pancreatic tissues in adult mice, which were studied 2 weeks post-injection.

Results Using the TUNEL assay, we demonstrated for the first time that in vivo injection of lentivirus shRNA Grb10 directly into the adult mouse pancreas induced apoptosis in both exocrine and endocrine (alpha and beta) cells. This effect was more pronounced in alpha cells. Levels of the pro-apoptotic protein BCL2-interacting mediator of cell death (BIM) in islets was higher in lentivirus shRNA Grb10 than in lentivirus shRNA scramble mice. In the apoptotic pathway, BIM initiates apoptosis signalling, leading to activation of the caspase cascade. We propose that, when complexed with GRB10, BIM is inactive. On activation by stress signalling or, in the present study, following injection of lentivirus shRNA Grb10 into pancreas, BIM becomes
\end{abstract}

Electronic supplementary material The online version of this article (doi:10.1007/s00125-011-2414-z) contains peer-reviewed but unedited supplementary material, which is available to authorised users.

B. Doiron $(\bowtie) \cdot$ W. Hu $\cdot$ L. Norton $\cdot$ R. A. DeFronzo

Diabetes Division, University of Texas

Health Science Center at San Antonio,

Mail Code 7886, 7703 Floyd Curl Drive,

San Antonio, TX 78299, USA

e-mail: doiron@uthscsa.edu

W. $\mathrm{Hu}$

Department of Metabolism,

General Hospital of Tianjin Medical University,

Tianjin, People's Republic of China unbound from GRB10 and activates the caspase cascade. Indeed, caspase-3 activity in islets was higher in the experimental than in the control group. Apoptosis induced by shRNA Grb10 resulted in a 34\% decrease in fasting plasma glucagon. Mice injected with shRNA Grb10 had improved glucose tolerance despite reduced insulin secretion compared with shRNA scramble control mice.

Conclusions/interpretation GRB10 is critically involved in alpha cell survival and, as a result, plays an important role in regulating basal glucagon secretion and glucose tolerance in adult mice.

Keywords Alpha cell apoptosis · Alpha cells · Apoptosis . Beta cells · Diabetes · Glucagon · Grb10 · Lentivirus

$\begin{array}{ll}\text { Abbreviations } \\ \text { AKT } & \begin{array}{l}\text { v-Akt murine thymoma viral oncogene } \\ \text { homologue }\end{array} \\ \text { BCL } & \text { B-cell leukaemia/lymphoma } \\ \text { BIM } & \text { BCL2-interacting mediator of cell death } \\ \text { GAPDH } & \text { Glyceraldehyde-3-phosphate dehydrogenase } \\ \text { GFP } & \text { Green fluorescent protein } \\ \text { GRB10 } & \text { Growth factor receptor-bound protein-10 } \\ \text { PI3K } & \text { Phosphatidylinositol 3-kinase } \\ \text { RAF-1 } & \text { v-Raf-1 murine leukaemia viral oncogene } \\ & \text { homologue 1 } \\ \text { shRNA } & \text { Small hairpin RNA }\end{array}$

\section{Introduction}

Type 2 diabetes mellitus results from an imbalance between insulin secretion and insulin resistance [1]. The insulin resistance involves multiple tissues, including liver and muscle [1, 2]. Overproduction of glucose by the liver, despite the presence of hyperinsulinaemia, is responsible for fasting hyperglycaemia, while impaired muscle glucose uptake 
primarily accounts for postprandial hyperglycaemia $[1,3,4]$. Type 2 diabetic patients are also characterised by hyperglucagonaemia and enhanced hepatic glucose production in response to glucagon $[3,5]$. Progression from normal glucose tolerance to impaired glucose tolerance to type 2 diabetes is characterised by a progressive decline in insulin secretion and plasma insulin concentration $[1,6-10]$ and a progressive rise in plasma glucagon concentration [3, 11, 12].

Beta cell mass is the result of the overall balance between beta cell proliferation (replication and neogenesis) and apoptosis. When the apoptosis rate exceeds the rate of beta cell proliferation, insulin secretion becomes inadequate and hyperglycaemia ensues [13]. While the loss of pancreatic beta cells in type 2 diabetes is well documented [14], the pathophysiological mechanism(s) responsible for the beta cell loss remain poorly defined. Recent studies have demonstrated that in diabetic individuals [15] alpha cell mass is increased, but the underlying molecular mechanisms responsible for the increase in alpha cell mass remain undefined. To date, no study has examined why alpha cell mass is increased whereas beta cell mass is decreased in the diabetic pancreas.

Early studies focused on the role of growth factor receptorbound protein-10 (GRB10) in the regulation of insulin sensitivity in peripheral tissues, primarily muscle $[16,17]$. GRB10 is an adaptor protein that inhibits tyrosine phosphorylation of the insulin/IGF-1/epidermal growth factor (EGF)/growth hormone and other growth factor receptors, thereby impairing downstream signalling pathways [17]. However, most previous GRB10 studies have been conducted in immortalised cell lines in vitro and it is not clear what role GRB10 plays in modulating insulin sensitivity in vivo. Moreover, a recent study using crystallography demonstrated that GRB10 binds to the insulin receptor far too weakly to have any significant physiological effect on insulin action [18]. These results raise questions about the proposed role of the GRB10 protein in the development of skeletal muscle insulin resistance, at least via any effect on insulin receptor phosphorylation $[16,17]$. Of note, GRB10 has been shown to be expressed in the rodent and human pancreas [19, 20]. However, no prior study has investigated the role of GRB10 in pancreatic tissues postnatally.

GRB10 has been implicated in apoptotic signalling [21], and it is highly concentrated in the mitochondria, an organelle involved in the regulation of apoptosis. GRB10 binds with a family of cell-survival-related partners including the IGF-1 receptor, v-raf-1 murine leukaemia viral oncogene homologue 1 (RAF-1), v-akt murine thymoma viral oncogene homologue (AKT) kinases, the p85 subunit of phosphatidylinositol 3kinase (PI3K), the 14-3-3 adaptor protein and the neuronal precursor cell-expressed developmentally downregulated 4 (NEDD4) ubiquitin ligase $[22,23]$. PI3K and AKT play an important role in beta cell survival [24-26]. Recently, it has been demonstrated that inhibition of the serine threonine kinase, RAF-1, in a beta cell line induces apoptosis in vitro [27]. Interestingly, GRB10 has been shown to participate in RAF-1-mediated cell survival signalling through inactivation of the pro-apoptotic protein B-cell leukaemia/ lymphoma-2 associated agonist of cell death (BAD) in a mitogen-activated protein kinase kinase (MEK)/extracellular signal-regulated kinase (ERK)- and PI3K/AKT-dependent manner [28].

Like retroviral vectors, lentivirus vectors do not encode any viral proteins. An advantage of lentivirus vectors is that they do not activate dendritic cells, like adenovirus vectors [29]. Furthermore, they can infect and integrate into both dividing and non-diving cells, they provide high transduction efficiency and sustained gene expression in vivo, they do not induce a significant host immune response [30-32] and, in contrast to adenovirus vectors, lentivirus vectors can be successfully re-administered [30]. Importantly, our method of viral vector injection in vivo into the adult murine pancreas also permits one to recreate the pathophysiology of a chronic disease that develops in adulthood and avoid the development of compensatory mechanisms that occur when a gene is deleted during embryonic development. This helps to obviate some of the paradoxical findings that have been reported with knockout models, i.e. normal/near-normal muscle insulin sensitivity in mice in which the insulin receptor is knocked out [33] and a homozygous null mutant for GLUT4 $\left(\mathrm{Glut4}^{-/-}\right)$that did not show a diabetic phenotype [34]. Using lentivirus small hairpin RNA (shRNA) silencing experiments, we have provided evidence for a critical role of GRB10 in alpha cell apoptosis. Specifically, we have shown, for the first time, that silencing of Grb10 in the adult mouse pancreas causes alpha cell apoptosis and reduced fasting plasma glucagon levels. To our knowledge, this is the first description of an anti-apoptotic molecule that regulates alpha cell survival in vivo and modulates circulating plasma glucagon levels. Given the important role of GRB10 in apoptotic mechanisms, we postulate that GRB10 is a mediator of apoptosis in the adult pancreas. Our technique, which allows us to deliver lentivirus shRNA specifically into the mouse pancreas in vivo, provides evidence that GRB10 is critically involved in alpha cell survival and, as a result, plays an important role in regulating basal glucagon secretion and glucose tolerance in adult mice.

\section{Methods}

Lentivirus construct We determined that the shRNA sequence 5'-GAACAACGATATTAACTCGTCCGTTCAA GAGACGGACGAGTTAATATCGTTGTTC-3' against the GRB10 mouse protein was able to effectively inhibit expression of Grb10 (Fig. 1a) in the islets and exocrine tissues (electronic supplementary material [ESM] Fig. 1). This shRNA sequence was introduced into a lentiviral vector under 

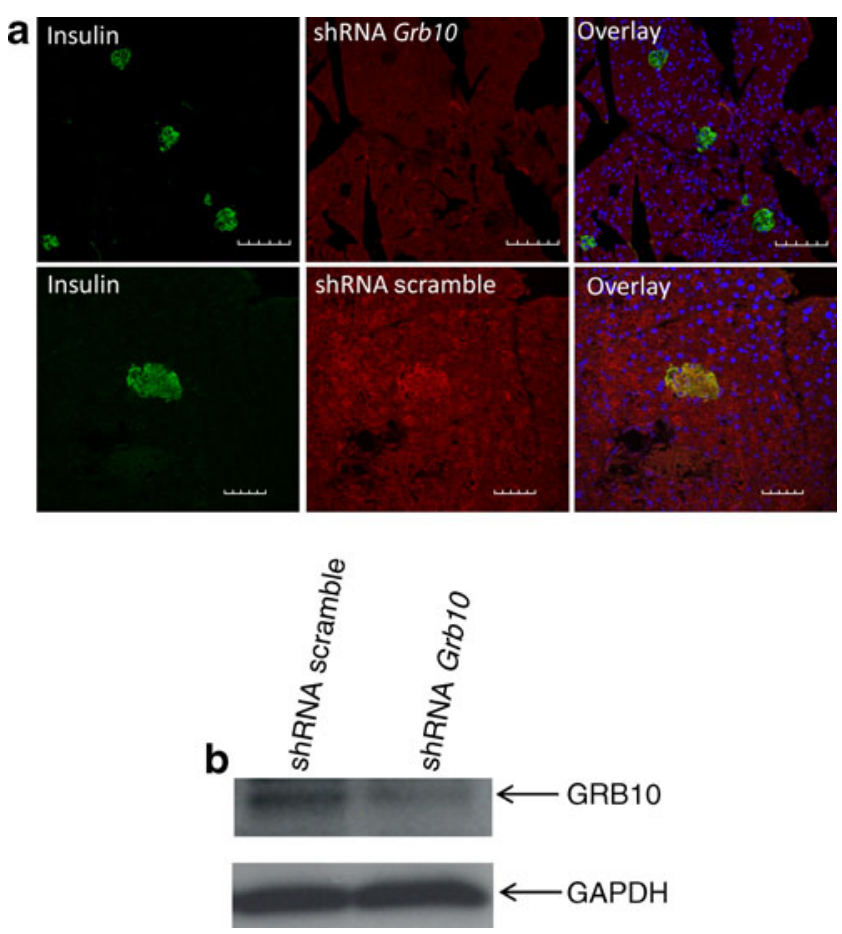

Fig. 1 Suppression of GRB10 in islets and exocrine tissues 2 weeks post-injection of lentivirus shRNA Grb10 via the pancreatic ductal route. a Insulin is shown in green and GRB10 in red. DAPI (blue) is used to identify nuclei. Lentivirus shRNA Grb10 histology scale bar length $100 \mu \mathrm{m}$ and lentivirus shRNA scramble histology scale bar length $50 \mu \mathrm{m}$. b Western blot of pancreatic tissues 2 weeks postinjection with lentivirus shRNA scramble and lentivirus shRNA Grb10 with anti-GRB10, stripped and then re-probed with antiGAPDH antibodies as a loading control. The figure is representative of five to six similar experiments. The western blot is representative of four individual samples in each experimental group. Six mice received the lentivirus shRNA Grb10 injection and five mice received the lentivirus shRNA scramble injection

the control of the $\mathrm{H} 1$ promoter. The polymerase III promoter $\mathrm{H} 1$ is active ubiquitously in all cells, because of the housekeeping function of polymerase III. The lentivirus shRNA Grb10 and shRNA scramble were generated by Welgen (Worcester, MA, USA).

Animal studies Male C57BL/6 mice (Charles River, Wilmington, MA, USA) 8 weeks of age were used for all experiments and were maintained on a diet of water and normal chow ad libitum. Animal protocols were approved by the University of Texas Health Science Center Animal Care Committee. Lentiviral shRNA constructs were introduced into the mouse pancreas via the intraductal route, as follows: a 32-gauge catheter (Braintree Scientific, Braintree, MA, USA) was inserted into the cystic duct through a small opening in the gallbladder. The catheter was then advanced into the common bile duct and secured in place with a slipknot of $0 / 0$ suture around the bile duct and catheter to prevent vector reflux into the liver. With a micro clamp placed around the sphincter of Oddi to avoid leakage of the vector into the duodenum, $100 \mu \mathrm{l}$ lentiviral vector expressing shRNA Grb10 or shRNA scramble at $1 \times 10^{8} \mathrm{TU} / \mathrm{ml}$ was slowly injected into the pancreatic duct through the catheter. At 1 day postinjection with lentiviral vector, the mice were injected i.p. daily with BrdU (Sigma-Aldrich, St Louis, MO, USA) in PBS at a dose of $50 \mu \mathrm{g} / \mathrm{g}$ body weight for 12 days. At 2 weeks post-lentiviral infection, the entire pancreas was removed for histological examination (see below). Figure 2 shows that, after $48 \mathrm{~h}$, injection of lentivirus coding for the green fluorescent protein (GFP) under the control of promoter cytomegalovirus specifically targeted the pancreatic tissues (ESM Fig. 2, for lentivirus H1 promoter-shRNA co-expressing GFP). The lentivirus-vector-produced GFP in vivo in the pancreas was not found in any other tissues in the body including heart, lung, liver, brain, leg muscle or kidney by histology or PCR (data not shown).

Intraperitoneal glucose tolerance test and insulin tolerance test Following an overnight fast, mice were weighed and injected i.p. with a bolus of glucose ( $2 \mathrm{~g} / \mathrm{kg}$ body weight). Blood glucose levels were determined from tail blood at 0 , $10,15,30,60$ and 120 min following glucose injection using an Ascensia Breeze 2 glucose meter (Bayer HealthCare, Mishawaka, IN, USA). Plasma insulin concentration was measured at $0,15,60$ and $120 \mathrm{~min}$ on $5 \mu \mathrm{l}$ (EDTA) samples using the mouse insulin Ultrasensitive ELISA (Alpco Diagnostics, Salem, NH, USA). Fasting plasma glucagon concentration was determined from $50 \mu \mathrm{l}$ plasma samples (EDTA, aprotinin 500 kIU [Trasylol; Bayer HealthCare, Pittsburgh, PA, USA] for every $1 \mathrm{ml}$ blood) using a mouse glucagon ELISA assay (Alpco Diagnostics). For the insulin tolerance test, following a $5 \mathrm{~h}$ fast, mice received an i.p. injection of $0.7 \mathrm{U} / \mathrm{kg}$ insulin (Novo Nordisk, Bagsvaerd, Denmark) in PBS.

Immunofluorescent and immunohistochemical analysis Adult mouse pancreatic tissues were fixed by immersion in phosphate buffer with $4 \%$ paraformaldehyde and $1 \%$
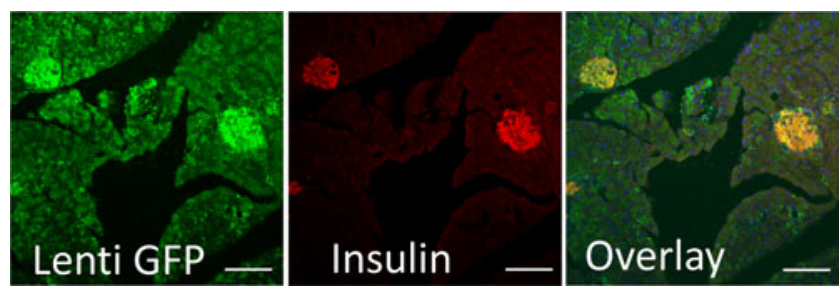

Fig. 2 In vivo injection of lentivirus (lenti) vector expressing GFP into the pancreatic duct. At $48 \mathrm{~h}$ post-injection, the pancreas was extracted for histological analysis. Insulin is shown in red and GFP in green. DAPI (blue) was used to identify the nucleus. Confocal laser microscopy was used for analysis. The $\gamma$ setting was modified because FITCconjugated squelch Texas Red conjugated on the overlay photo. The figure is representative of five similar experiments. Scale bar length $100 \mu \mathrm{m}$ 
glutaraldehyde overnight at $4{ }^{\circ} \mathrm{C}$ and subsequently embedded with Tissue-Tek OCT compound for cryostat sectioning. The following primary antibodies were used: anti-somatostatin (G-10), anti-Ki-67 (M-19), anti-glucagon (K79bB10), antiGRB10 (K-20), anti-insulin A (C-12), anti-BCL2-interacting mediator of cell death (BIM) (M-20) and anti-amylase (C-20) antibodies and control rabbit IgG (Santa Cruz Biotechnology, Santa Cruz, CA, USA). The activity of caspase-3 was measured with an antibody (Cell Signaling Technology, Danvers, MA, USA) that detects endogenous levels of the large fragment $(17 / 19 \mathrm{kDa})$ of activated caspase- 3 resulting from cleavage next to Asp175. For proliferation studies, pancreatic tissues were stained with either Ki-67 (M-19) antibody (Santa Cruz) or with rat monoclonal BrdU antibody (Abcam, Cambridge, MA, USA). Antigen retrieval was performed for Ki-67 and BrdU antibodies by boiling sections for $10 \mathrm{~min}$ in $10 \mathrm{mmol} /$ 1 citrate buffer followed by cooling for $30 \mathrm{~min}$ to room temperature. Nuclei were counterstained with DAPI (Vector Laboratories, Burlingame, CA, USA). The fluorescent secondary antibodies used included donkey anti-goat-fluorescein, goat anti-mouse-fluorescein, goat anti-rabbit Texas Red and donkey anti-goat Texas Red (Santa Cruz). The beta cell area represents the surface area of cells staining positively for insulin immunostaining divided by the total pancreatic surface scanned with an Olympus FV-1000 laser scanning confocal microscope. The insulin-positive and total pancreatic areas were quantified with Image J (National Institutes of Health, Bethesda, MD, USA). Beta cell mass was calculated as beta cell area multiplied by pancreatic wet weight. At least three mice were analysed per condition. TUNEL assay was measured with the in situ Cell Death Detection Kit, TMR Red (Roche Diagnostics, Indianapolis, IN, USA). Quantification of pancreatic transduction by lentiviral GFP vector following intraductal injection was obtained by visualising the GFP expression area of the pancreas performed in four sections per animal, separated by $200 \mu \mathrm{m}$. To quantify the percentage of the area expressing GFP, images were analysed with ImageJ software to determine the GFP-positive area of each image. Briefly, images were subjected to thresholding using a GFP-negative control section from untreated pancreatic tissue as a reference for background GFP level. The percentage of area with brightness values equal to or exceeding the threshold value within each image was then calculated and averaged for all four images per animal. Pancreatic tissue was stained with haematoxylin and eosin to look for evidence of inflammation (pancreatitis) at day 2 and day 14 post-injection of the lentivirus.

$R N A$ extraction and real-time PCR For comparison, we examined the expression of GRB10 in human skeletal muscle, adipose tissue and pancreas. Total RNA was extracted from approximately $20-30 \mathrm{mg}$ of frozen skeletal muscle and fat tissue using TRIzol reagent (Invitrogen, Carlsbad, CA, USA).
Human pancreatic RNA was obtained from Clontech (cat. \#636577). The integrity of each RNA sample was confirmed post-extraction using denaturing (glyoxal) agarose gel electrophoresis. Reverse transcription was carried out with $0.5 \mu \mathrm{g}$ total RNA using the ImProm II reverse transcription system (Promega, Madison, WI, USA) according to the manufacturer's instructions. Real-time quantitative PCR was then performed using $2 \mu \mathrm{l}$ cDNA with a primer and 5'-terminal 6-carboxyfluorescein (FAM)-labelled TaqMan probe mix from Applied Biosystems (assay ID Hs00193409; Foster City, CA, USA). Relative expression values were calculated from a standard curve, which consisted of a twofold dilution series from a pooled sample of each cDNA, and they were normalised to $18 \mathrm{~S}$ ribosomal RNA. Data for PEPCK (also known as PCK1) were normalised to GAPDH mRNA levels, which were not affected by shRNA delivery.

Western blot For western blots, equal amounts of total protein were separated on a $10 \%$ and $15 \%$ SDS/PAGE and transferred onto nitrocellulose membranes. Membranes were then blocked with $5 \%$ non-fat milk in $0.1 \%$ Tris buffered saline (TBS) Tween-20 and probed with specific antibodies against GRB10 (Santa Cruz), cleaved caspase-3 (Cell Signaling Technology) and glyceraldehyde-3-phosphate dehydrogenase (GAPDH; G9545, Sigma-Aldrich). Membranes were then incubated with horseradish peroxidaseconjugated secondary antibody (NA934) and developed with a chemiluminescent reagent (Amersham Bioscience, GE Healthcare, Pittsburgh, PA, USA).

Statistical analysis Results are presented as mean \pm SEM. Statistical comparisons were performed with Student's unpaired $t$ test or one-way ANOVA, as appropriate. Results were considered to be statistically significant when $p<0.05$.

\section{Results}

GRB10 localisation in the pancreas To determine the localisation of GRB10 in the adult mouse pancreas, we performed confocal laser microscope studies. Co-staining of pancreatic sections of C57BL/6 mice with a monoclonal anti-insulin antibody and a polyclonal anti-GRB10 antibody revealed that GRB10 protein is present in the pancreatic endocrine and exocrine tissues (Fig. 3). Overlay of the GRB10 with other antibody-staining images revealed co-localization of GRB10 in endocrine (alpha, beta and delta) and exocrine (amylase and ductal cells) cells (Fig. 3). By quantitative RT-PCR, we demonstrated that GRB10 also is expressed in human pancreas. Of the tissues examined, GRB10 mRNA levels were highest in the human pancreas, being almost twice as abundant as in human skeletal muscle (ESM Fig. 3). 

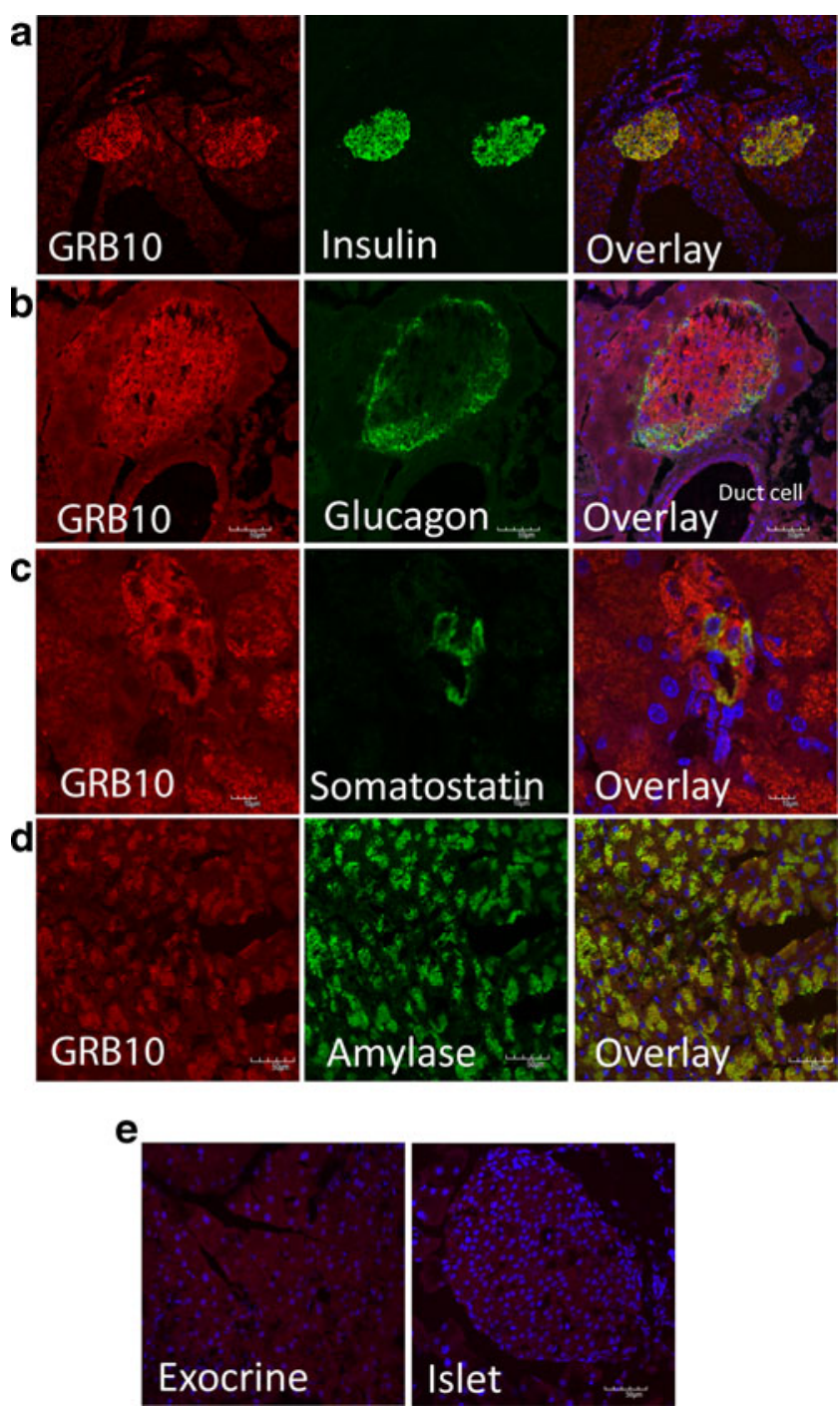

Fig. 3 Mouse pancreatic tissues. a Insulin is shown in green and GRB10 in red. DAPI (blue) is used to identify nuclei in all panels. b Glucagon is shown in green and GRB10 in red (scale bar, $50 \mu \mathrm{m}$ ) c Somatostatin is shown in green and GRB10 in red (scale bar, $10 \mu \mathrm{m}$ ). d Amylase is shown in green and GRB10 in red (scale bar, $50 \mu \mathrm{m}$ ). The overlay represents DAPI/green/red. e A rabbit isotype control at the same concentration as the GRB10 antibody and exposure time (scale bar, $50 \mu \mathrm{m}$ ). The figure is representative of ten similar experiments

Lentiviral vector injection into the mouse pancreas We developed a technique that allows injection of the viral vector in vivo directly into the pancreas in adult mice (see 'Animal studies' under experimental procedures) and demonstrated that, $48 \mathrm{~h}$ post-injection, the lentivirus vector produced GFP in vivo in the pancreas (Fig. 2; see also ESM Fig. 2) and not in any other tissues in the body. Quantitative morphometric analysis of pancreatic transduction by the lentivirus vector, based on GFP production, showed that $60 \%$ of the tissue produced GFP. Importantly, expression was detected in the pancreas even after 14 days. Lentiviral-vectorconstructed shRNA targeting Grb10 was injected in vivo via the intraductal pancreatic route to target the pancreatic tissues in 8-week-old adult mice $(n=6)$, which were studied 2 weeks post-injection. Control mice $(n=5)$ were injected with a lentivirus shRNA scramble using the same technique. BrdU, a marker of cell proliferation, was administered daily for 2 weeks by i.p. injection to both experimental and control groups. Following the lentiviral vector injection, activity, food intake (daily food intake in $\mathrm{g}$ over the 14 days post-injection: mice shRNA scramble $5.4 \pm 0.3 ; n=5$ vs mice shRNA Grb10 5.9 $\pm 0.5 ; n=6$ ) and weight gain were similar in the shRNA Grb10 and shRNA scramble groups (ESM Fig. 4). No diarrhoea was observed in the control and experimental groups after the lentivirus injection.

In the experimental group injected with lentivirus shRNA Grb10, histological examination demonstrated the suppression of GRB10 protein production using western blot (Fig. 1b). In the experimental group injected with shRNA Grb10, we did not observe any increase in cell proliferation with antibodies against BrdU or Ki-67 (data not shown). In contrast, using the TUNEL assay, we demonstrated for the first time that in vivo injection of lentivirus shRNA Grb10 directly into the adult mouse pancreas induced apoptosis in both exocrine and endocrine tissues (Fig. 4a-c). The major apoptotic physiological effect of shRNA Grb10 in the adult pancreas was observed against the alpha cells (Fig. 4d; ESM Fig. 5) and was associated with a 34\% decrease in fasting plasma glucagon concentration ( $86 \pm 12$ vs $129 \pm 7 \mathrm{ng} / \mathrm{l}, p<$ $0.05)$. However, as pointed out previously [35], the in vivo histological TUNEL assay for apoptosis of pancreatic beta cells is limited by the small number of these cells that are undergoing apoptosis at any given time. Similarly, it is technically difficult to accurately quantify in vivo the number of glucagon cells that are undergoing apoptosis. Another obstacle that complicates the quantification of apoptosis in vivo is the speed at which cells die and disappear. The time from initiation of apoptosis to its completion can be as short as $2-3 \mathrm{~h}$ [36]. Another challenge in quantifying apoptosis is related to the asynchronous nature of the apoptotic process [37], and this could explain why the number of beta cells staining for caspase-3 activity exceeds the number of TUNEL-assay-positive beta cells [37-39]. Nonetheless, our results clearly demonstrate that shRNA Grb10 induced alpha and beta cell apoptosis in vivo within the limits of the methods employed to quantify apoptosis. Interestingly, the level of the pro-apoptotic protein BIM in islets was higher in lentivirus shRNA Grb10 mice compared with lentivirus shRNA scramble mice (Fig. 5; ESM Figs 6 and 7). In addition, the caspase- 3 activity in islets was higher in the mice injected with lentivirus shRNA Grb10 compared with the control shRNA scramble group (Fig. 5). Caspase-3 and BIM represent steps that precede the death of cells and would not be expected to modify the structure of the cell. Other investigators have observed the same phenomenon, i.e. 

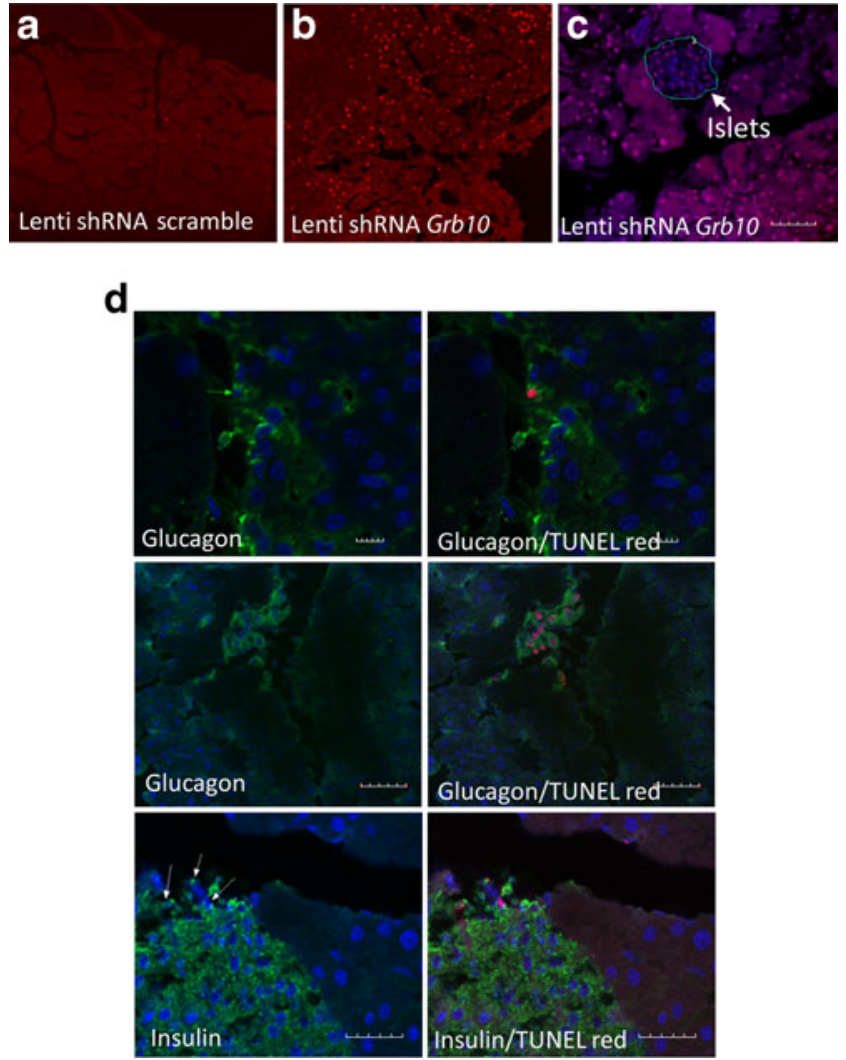

Fig. 4 TUNEL assay of pancreatic tissues 2 week post-injection with lentivirus shRNA Grb10 and scramble. The in situ Cell Death Detection Kit TMR Red (Roche Diagnostics) was used for the TUNEL assay. a Pancreatic tissue injected with lentivirus shRNA scramble shows no apoptotic cells with TUNEL assay. b Pancreatic tissue injected with lentivirus shRNA Grb10 demonstrates apoptotic cells. c Pancreatic tissue injected with lentivirus shRNA Grb10 shows apoptotic cells in the islets (scale bar, $50 \mu \mathrm{m}$ ). DAPI (blue) was used to identify the nuclei. The figure is representative of an area of the pancreas examined in ten sections per animal, separated by $200 \mu \mathrm{m}$. Six mice received the lentivirus shRNA Grb10 injection and five mice received the lentivirus shRNA scramble injection. $\mathbf{d}$ TUNEL assay of pancreatic tissues showing lentivirus shRNA Grb10-induced alpha cell apoptosis (scale bars, $10 \mu \mathrm{m}$ to $50 \mu \mathrm{m}$ ) and beta cell apoptosis (scale bar, $20 \mu \mathrm{m}$ ) Antibody against glucagon and insulin is shown in green. The TUNEL assay employed red antibody. DAPI (blue) is used to identify the nuclei. The figure is representative of different areas of the pancreas examined in ten sections per animal, separated by $200 \mu \mathrm{m}$. Six mice received the lentivirus shRNA Grb10 injection and five mice received the lentivirus shRNA scramble injection. Lenti, lentivirus

an increase in caspase- 3 activity without any change in cell architecture [38, 39]. One can also have a situation with increased caspase-3 activity and/or BIM production in which the apoptotic cascade is fully engaged and the cellular architecture is distorted (ESM Figs 6 and 7). There was no effect of the lentivirus shRNA Grb10 on fasting plasma insulin concentration, which was similar in both groups $(492 \pm$ $658 \mathrm{pmol} / 1$ in shRNA Grb10 vs $669 \pm 83 \mathrm{pmol} / 1$ in shRNA scramble, $p=\mathrm{NS}$ ). There was no effect of the lentivirus shRNA Grb10 on insulin tolerance test results compared with the lentivirus shRNA scramble group (ESM Fig. 8). Mice injected with shRNA Grb10 had improved i.p. glucose tolerance test results (Fig. 6b) despite reduced insulin secretion (Fig. 6c) compared with shRNA scramble control mice.

\section{Discussion}

It has been demonstrated previously that GRB10 is an antiapoptotic protein in cell lines in vitro [25]. We demonstrate, for the first time in vivo, the important role of GRB10 in the survival of pancreatic cells. The Src-homology 2 (SH2)- and pleckstrin homology $(\mathrm{PH})$-domain-containing protein, GRB10, has been shown to be produced in the rodent and human pancreas $[19,20]$ but, to date, no study has delineated the precise distribution of GRB10 expression in the pancreas. We show here that $G r b 10$ is highly expressed in adult mouse pancreatic beta cells (insulin) and at lower levels in alpha cells (glucagon) and delta cells (somatostatin), as well as in the exocrine pancreas (amylase and ductal cells) (Fig. 3). We have also demonstrated that Grblo is highly expressed in the human pancreas compared with insulin-sensitive tissues, skeletal muscle and fat (ESM Fig. 3). We demonstrate that silencing of Grb10 in the adult mouse pancreas causes alpha cell apoptosis and reduced fasting plasma glucagon levels (Figs 4 and 6a; ESM Fig. 5). To our knowledge, this is the first description of an anti-apoptotic molecule that regulates alpha cell survival in vivo and modulates circulating plasma glucagon levels.

Recently, overexpression of Grb10 in the HEK293 cell line has been shown to inhibit the activity of BIM, a proapoptotic protein [40]. BIM is regulated at both the transcriptional and post-translational levels [41]. In the present study, the level of the pro-apoptotic protein BIM in islets was higher in mice receiving lentivirus shRNA Grb10 compared with lentivirus shRNA scramble mice (Fig. 5). In the apoptotic pathway, the protein BIM initiates apoptosis signalling by binding to the B-cell leukaemia/lymphoma 2 (BCL-2)-like pro-survival proteins (BCL-2), BCL2-like protein 1 (BCL-XL), BCL-W, myeloid cell leukaemia 1 (MCL-1) and Bcl gene A1, thereby releasing BAX and/or BAK, which causes a decrease in the mitochondrial outer membrane potential, release of cytochrome $c$ and activation of the caspase cascade [42]. We hypothesise that, when complexed with GRB10, BIM is inactive. Upon cellular activation by stress signalling or, as in the present study, following injection of lentivirus shRNA Grb10 into the pancreas, BIM becomes unbound from GRB10 and causes activation of BAX-BAK, thereby inducing apoptosis by activating the caspase cascade. Indeed, as demonstrated in Fig. 5, the activity of caspase-3 in islets was higher in the experimental group compared with the control group. 

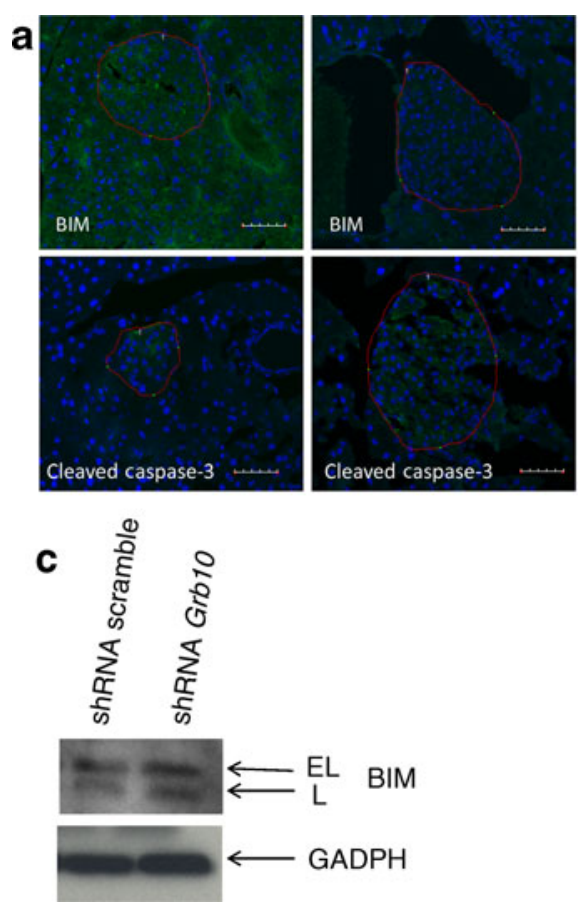

Fig. 5 Production of pro-apoptotic protein BIM and cleaved caspase-3 activity in islets 2 weeks post-injection of lentivirus shRNA scramble (a) and lentivirus shRNA Grb10 (b). Scale bars $50 \mu \mathrm{m}$. The figure is representative of different areas of the pancreas examined in seven sections per animal, separated by $200 \mu \mathrm{m}$. Six mice received the lentivirus shRNA Grb10 injection and five mice received the lentivirus shRNA scramble injection. c, d Western blots of pancreatic tissues

The lentivirus shRNA Grb10 also induced exocrine cell apoptosis (Fig. 4; ESM Figs 9 and 10). However, there was no histological evidence of inflammation and pancreatic wet weight, as well as body weight gain, was similar in the two experimental groups (ESM Figs 4 and 11). Consistent with this, lentivirus has been shown not to induce inflammation, the hallmark of an immune response, at the site of injection [30]. b
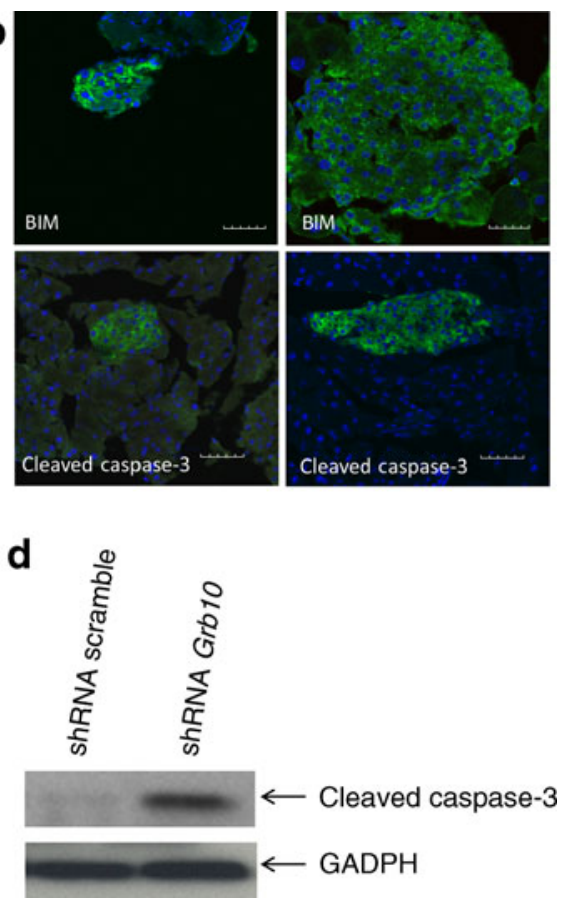

2 weeks post-injection with lentivirus shRNA scramble and lentivirus shRNA Grb10 with anti-cleaved caspase-3, stripped and then reprobed with anti-GAPDH antibodies as a loading control. The western blot is representative of four individual samples in each experimental group. Two distinct isoforms of BIM are shown (c): L, BIM long; and EL, BIM extra long

At day 7 and 14 days post-injection, the amylase level was normal. Therefore, apoptosis of the exocrine tissues in the experimental group injected with the lentivirus shRNA Grb10 did not cause symptoms indicative of chronic pancreatitis. As the exocrine pancreas constitutes more than $95 \%$ of total pancreatic tissue [43], our results indicate that, if only a small percentage a

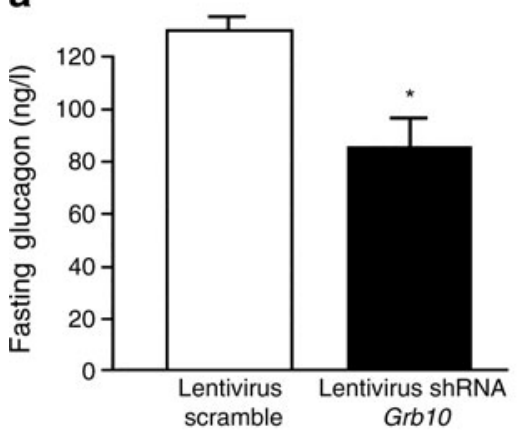

b

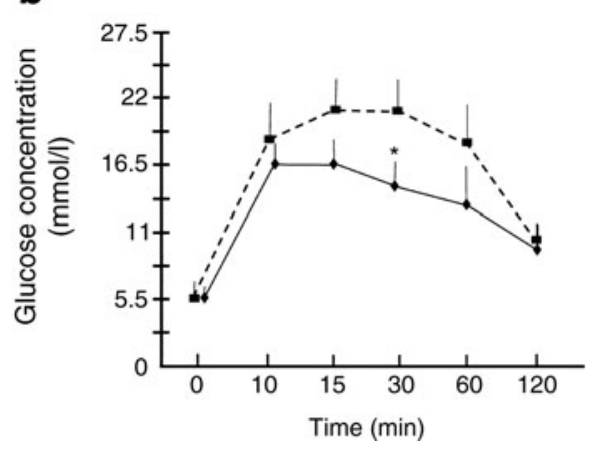

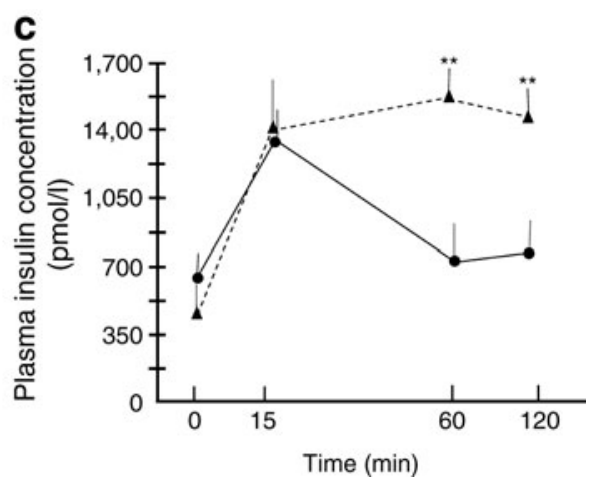

Fig. 6 a Fasting plasma glucagon concentration 2 weeks postinjection of lentivirus shRNA Grb10. Blood samples were collected after a 14 h overnight fast. Plasma glucagon was measured using the Alpco immunoassay kit for glucagon. ${ }^{*} p<0.05$ lentivirus shRNA scramble $(n=5)$ vs lentivirus shRNA Grb10 $(n=6)$. b Blood glucose concentration during the i.p. glucose tolerance test. Squares, lentivirus
shRNA scramble; diamonds, lentivirus shRNA Grb10. ${ }^{*} p<0.05$ lentivirus scramble $(n=5)$ vs lentivirus shRNA $\operatorname{Grb10}(n=6)$. c Plasma insulin concentration during the i.p. glucose tolerance test. Triangles, lentivirus shRNA scramble; circles, lentivirus shRNA Grb10. $* * p<0.01$ lentivirus shRNA scramble $(n=5)$ vs lentivirus shRNA $\operatorname{Grb10}(n=6)$ 
of exocrine tissue undergoes apoptosis, the remaining exocrine tissue is capable of compensating for any tissue that is lost.

In mice the majority of cells within the islets are beta cells (approximately 65-75\%); glucagon-secreting alpha cells represent less than $20 \%$ of the individual islet area [44]. Therefore, one might expect that loss of alpha cells via apoptosis would have a greater physiological impact on glucose homeostasis than the loss of an equivalent or even greater number of beta cells. Indeed, this is precisely what has been observed in partially pancreatectomised patients [45-47]. Thus, despite a modest decrease in fasting glucagon concentration and a severe reduction in the plasma insulin and C-peptide response to glucose in partially pancreatectomised individuals, glucose tolerance and insulin action are not adversely affected [47]. These results suggest that the decrease in plasma glucagon concentration after subtotal pancreatectomy may be associated with enhanced insulin sensitivity and are consistent with previously published results from our group that demonstrated that a physiological elevation in plasma glucagon concentration in individuals with normal glucose tolerance for as little as 3 days induces moderate to severe insulin resistance [48]. In the present study, injection of lentivirus shRNA Grb10 directly into the pancreas produced a similar phenotype with decreased fasting glucagon concentration (Fig. 6a) and an improved glucose tolerance test despite a decrease in insulin secretion (Fig. 6b). Thus, simultaneous apoptosis of both alpha and beta cells, induced by lentivirus shRNA Grb10 (Fig. 4), explains the reduction in fasting plasma glucagon concentration and, consequently, improved i.p. glucose tolerance despite a decrease in insulin secretion.

The reduction in fasting plasma glucagon concentration with maintained fasting plasma insulin level despite the $40 \%$ reduction in beta cell mass (ESM Fig. 12) was not associated with a decline in fasting plasma glucose concentration. There was no effect of the lentivirus shRNA Grb10 on insulin tolerance test compared with the lentivirus shRNA scramble group (ESM Fig. 8). Because, in rodents, hepatic glucose production under fasting conditions is primarily derived from gluconeogenesis [49] and PEPCK is the rate-limiting enzyme for gluconeogenesis, we measured the Pepck mRNA level in the liver. No difference in Pepck mRNA expression was observed in mice injected with shRNA Grb10 or shRNA scramble. The maintenance of normal hepatic Pepck gene expression/activity in mice injected with lentivirus shRNA Grb10 would be expected to maintain gluconeogenesis and basal hepatic glucose production, thus preventing any decline in fasting plasma glucose concentration. Although not measured in the present study, the maintenance of normal circulating catecholamine levels could also prevent the development of hypoglycaemia caused by glucagon deficiency [50].

In summary, we demonstrate for the first time that lentivirus shRNA Grb10 injected directly into the mouse pancreas induces apoptosis of both endocrine and exocrine cells. These findings represent the first demonstration that GRB10 is an anti-apoptotic protein in vivo. The apoptosis induced by shRNA Grb10 results in a decrease in fasting plasma glucagon concentration and improved i.p. glucose tolerance despite a decrease in glucose-stimulated insulin secretion. Our work provides a novel model to study both the apoptotic and anti-apoptotic signalling mechanisms of GRB10 in the pancreas. We anticipate that this will provide novel insights about the basic cellular/molecular mechanisms that regulate pancreatic beta and alpha cell mass and could have important implications for the pathogenesis of type 2 diabetes, which is characterised by increased glucagon secretion/alpha cell mass and reduced insulin secretion/ beta cell mass [1].

Funding Images were generated in the Core Optical Imaging Facility which is supported by the University of Texas Health Science Center at San Antonio (UTHSCSA), National Institutes of Health (NIH)-National Cancer Institute (NCI) P30 CA54174 (Cancer Therapy \& Research Center (CTRC) at UTHSCSA) and NIH-National Institute on Aging (NIA) P01AG19316.

Duality of interest The authors declare that there is no duality of interest associated with this manuscript.

Contribution statement $\mathrm{BD}$ designed the research and the experimental method of lentivirus injection to target the pancreas in vivo. BD performed animal research data analysis and histology, analysed data and wrote and edited the manuscript. WH performed the western blot and histology, analysed data and edited the manuscript. LN performed RT-PCR, analysed data and contributed to the manuscript. RAD analysed the data and wrote the manuscript. All authors approved the final version to be published.

\section{References}

1. DeFronzo RA (2009) Banting Lecture. From the triumvirate to the ominous octet: a new paradigm for the treatment of type 2 diabetes mellitus. Diabetes 58:733-795

2. Bajaj M, DeFronzo RA (2003) Metabolic and molecular basis of insulin resistance. J Nucl Cardiol 10:311-323

3. Matsuda M, DeFronzo RA, Glass L et al (2009) Glucagon doseresponse curve for hepatic glucose production and glucose disposal in type 2 diabetic patients and normal individuals. Metabolism 51:1111-1119

4. Edgerton DS, Johnson KM, Cherrington AD (2009) Current strategies for the inhibition of hepatic glucose production in type 2 diabetes. Front Biosci 14:1169-1181 
5. Dunning BE, Gerich JE (2007) The role of alpha-cell dysregulation in fasting and postprandial hyperglycemia in type 2 diabetes and therapeutic implications. Endocr Rev 28:253283

6. Gastaldelli A, Ferrannini E, Miyazaki Y, Matsuda M, DeFronzo RA (2004) Beta-cell dysfunction and glucose intolerance: results from the San Antonio metabolism (SAM) study. Diabetologia 47:31-39

7. Ferrannini E, Gastaldelli A, Miyazaki Y, Matsuda M, DeFronzo RA (2005) Beta-cell function in subject spanning the range from normal glucose tolerance to overt diabetes: a new analysis. J Clin Endocrinol Metab 90:493-500

8. Abdul-Ghani M, Jenkinson C, Richardson D, Tripathy D, DeFronzo RA (2006) Insulin secretion and action in subjects with impaired fasting glucose and impaired glucose tolerance: results from the Veterans Administration Genetic Epidemiology Study. Diabetes 55:1430-1435

9. Bergman RN, Finegood DT, Kahn SE (2002) The evolution of beta-cell dysfunction and insulin resistance in type 2 diabetes. Eur $\mathrm{J}$ Clin Invest 32:35-45

10. Reaven GM, Hollenbeck CB, Chen YD (1989) Relationship between glucose tolerance, insulin secretion, and insulin action in non-obese individual with varying degrees of glucose tolerance. Diabetologia 32:52-55

11. Baron AD, Schaeffer L, Shragg P, Kolterman OG (1987) Role of hyperglucagonemia in maintenance of increased rates of hepatic glucose output in type II diabetics. Diabetes 36:274283

12. Unger RH, Aguilar-Parada E, Muller WA, Eisentraut AM (1970) Studies of pancreatic alpha cell function in normal and diabetic subjects. J Clin Invest 49:837-848

13. Butler PC, Meier JJ, Butler AE, Bhushan A (2007) The replication of beta cells in normal physiology, in disease and for therapy. Nat Clin Pract Endocrinol Metab 3:758-768

14. de Koning EJ, Bonner-Weir S, Rabelink TJ (2008) Preservation of beta-cell function by targeting beta-cell mass. Trends Pharmacol Sci 29:218-227

15. Yoon KH, Ko SH, Cho JH et al (2003) Selective beta-cell loss and alpha-cell expansion in patients with type 2 diabetes mellitus in Korea. J Clin Endocrinol Metab 88:2300-2308

16. O’Neill TJ, Rose DW, Pillay TS, Hotta K, Olefsky JM, Gustafson TA (1996) Interaction of a GRB-IR splice variant (a human GRB10 homolog) with insulin and insulin-like growth factor I receptors. Evidence for a role in mitogenic signaling. J Biol Chem 271: 22506-22513

17. Holt LJ, Siddle K (2005) Grb10 and Grb14: enigmatic regulator of insulin action - and more? Biochem J 388:393-406

18. Depetris RS, Wu J, Hubbard SR (2009) Structural and functional studies of the Ras-associating and pleckstrin-homology domains of Grb10 and Grb14. Nat Struct Mol Biol 16:833840

19. McCann JA, Zheng H, Islam A, Goodyer CG, Polychronakos C (2001) Evidence against Grb10 as the gene responsible for Silver-Russel syndrome. Biochem Biophys Res Commun 286:943-948

20. Frantz JD, Giorgetti-Peraldi S, Ottinger EA, Human SSE, Shoelson SE (1997) Human GRB-IRbeta/GRB10: splice variants of an insulin and growth factor receptor-binding protein with $\mathrm{PH}$ and SH2 domains. J Biol Chem 272:2659-2667

21. Nantel A, Huber M, Thomas DY (1999) Localization of endogenous Grb10 to the mitochondria and its interaction with the mitochondria-associated Raf-1 pool. J Biol Chem 274:3571935724

22. Riedel H (2004) Grb10 exceeding the boundaries of a common signaling adapter. Front Biosci 9:603-618
23. Urschel S, Bassermann F, Bai RY, Munch S, Peschel C, Duyster J (2005) Phosphorylation of grb10 regulated its interaction with 14-3-3. J Biol Chem 280:16987-16993

24. Hashimoto N, Kido Y, Uchida T et al (2006) Ablation of PDK1 in pancreatic beta cells induces diabetes as a result of loss of beta cell mass. Nat Genet 38:589-593

25. Lingohr MK, Dickson LM, McCuaig JF, Hugl SR, Twardzik DR, Rhodes CJ (2002) Activation of IRS-2-mediated signal transduction by IGF-1, but not TGF-alpha or EGF, augments pancreatic beta-cell proliferation. Diabetes 51:966-976

26. Hennige AM, Burks DJ, Ozcan U et al (2003) Upregulation of insulin receptor substrate-2 in pancreatic beta cells prevents diabetes. J Clin Invest 112:1521-1532

27. Alejandro EU, Johnson JD (2008) Inhibition of Raf-1 alters multiple downstream pathways to induce pancreatic beta-cell apoptosis. J Biol Chem 283:2407-2417

28. Kebache S, Ash J, Annis MG et al (2007) Grb10 and active Raf-1 kinase promote Bad-dependent cell survival. J Biol Chem 282:21873-21883

29. Woodley DT, Keene DR, Atha T et al (2004) Intradermal injection of lentivirus vectors corrects regenerated human dystrophic epidermolysis bullosa skin tissue in vivo. Mol Ther $10: 318-326$

30. Kafri T, Blomer U, Peterson DA, Gage FH, Verma IM (1997) Sustained expression of genes delivered directly into the liver and muscle by lentiviral vectors. Nat Genet 17:314-317

31. Naldini L, Blomer U, Gage FH, Trono D, Verma IM (1996) Efficient transfer, integration, and sustained long-term expression of the transgene in adult rat brains injected with a lentiviral vector. Proc Nat Acad Sci USA 93:11382-11388

32. Amado RG, Chen IS (1999) Lentiviral vectors - the promise of gene therapy within reach? Science 285:674-676

33. Bruning JC, Michael MD, Winnay JN et al (1988) A musclespecific insulin receptor knockout exhibits features of the metabolic syndrome of NIDDM without altering glucose tolerance. Mol Cell 2:559-569

34. Katz EB, Stenbit AE, Hatton K, DePinho R, Charron MJ (1995) Cardiac and adipose tissues abnormalities but not diabetes in mice deficient in GLUT4. Nature 377:151-155

35. Meier JJ, Bhushan A, Butler AE, Rizza RA, Butler PC (2005) Sustained beta cell apoptosis in patients with long-standing type 1 diabetes: indirect evidence for islets regeneration? Diabetologia 48:2221-2228

36. Bursch W, Kleine L, Tenniswood M (1990) The biochemistry of cell death by apoptosis. Biochem Cell Biol 68:1071-1074

37. Collins JA, Schandi CA, Young KK, Vesely J, Willingham MC (1997) Major DNA fragmentation is a late event in apoptosis. J Histochem Cytochem 45:923-934

38. Woo M, Hakem R, Soengas MS et al (1998) Essential contribution of caspase 3/CPP32 to apoptosis and its associated nuclear changes. Genes Dev 12:806-819

39. Oberhammer F, Wilson JW, Dive C et al (1993) Apoptotic death in epithelial cells: cleavage of DNA to $300 \mathrm{and} /$ or $50 \mathrm{~kb}$ fragments prior to or in the absence of internucleosomal fragmentation. EMBO J 12:3679-3684

40. Hu ZQ, Zhang JY, Ji CN, Xie Y, Chen JZ, Mao YM (2010) Grb10 interacts with Bim L and inhibits apoptosis. Mol Biol Rep 37:3547-3552

41. Chipuk JE, Moldoveanu T, Llambi F, Parsons MJ, Green DR (2010) The BCL-2 family reunion. Molecular Cell 37:299-310

42. Willis SN, Fletcher JI, Kaufmann T et al (2007) Apoptosis initiated when $\mathrm{BH} 3$ ligands engage multiple $\mathrm{Bcl}-2$ homologs, not Bax or Bak. Science 315:856-859

43. Brannon PM (1990) Adaptation of the exocrine pancreas to diet. Annu Rev Nutr 10:85-105 
44. Brissova M, Fowler MJ, Nicholson WE et al (2005) Assessment of human pancreatic islet architecture and composition by laser scanning confocal microscopy. J Histochem Cytochem 53:1087-1097

45. Schrader H, Menge BA, Breuer TG et al (2009) Impaired glucoseinduced glucagon suppression after partial pancreatectomy. J Clin Endocrinol Metab 94:2857-2863

46. Ahren B, Andren-Sandberg A (1993) Capacity to secrete islet hormones after subtotal pancreatectomy for pancreatic cancer. Eur J Surg 159:223-227

47. Magnusson J, Bengmark S, Tranberg KG (1990) Reduced insulin secretion by subtotal pancreatectomy: preservation of insulin sensitivity and glucose tolerance in postoperative patients. Scand J Gastroenterol 25:669-675

48. Del Prato S, Castellino P, Simonson DC, DeFronzo RA (1987) Hyperglucagonemia and insulin-mediated glucose metabolism. J Clin Invest 79:547-556

49. Pilkis SJ, Granner DK (1992) Molecular physiology of the regulation of hepatic gluconeogenesis and glycolysis. Annu Rev Physiol 54:379-406

50. Koerker DJ, Halter JB (1982) Glucoregulation during insulin and glucagon deficiency: role of catecholamines. Am J Physiol 243: E225-E233 